\title{
Probing vortex-shedding at high frequencies in flows past confined microfluidic cylinders using high-speed microscale particle image velocimetry ${ }^{\circledR}$
}

Cite as: Phys. Fluids 31, 102001 (2019); https://doi.org/10.1063/1.5111817

Submitted: 30 May 2019 . Accepted: 09 September 2019 . Published Online: 02 October 2019

Shigang Zhang (D), Neil Cagney (D), Stavroula Balabani (D), Carolina P. Naveira-Cotta (D), and Manish K. Tiwari (i)

\section{COLLECTIONS}

Paper published as part of the special topic on Selected Articles from the 2018 Micro and Nano Flows Conference Note: This paper is part of the Special Issue from the 2018 Micro and Nano Flows Conference.

EP This paper was selected as an Editor's Pick

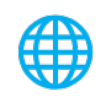

\section{ARTICLES YOU MAY BE INTERESTED IN}

Formation and turbulent breakdown of large-scale vortical structures behind an obstacle in a channel at moderate Reynolds numbers

Physics of Fluids 31, 104104 (2019); https://doi.org/10.1063/1.5120611

Modeling the interplay between the shear layer and leading edge suction during dynamic stall

Physics of Fluids 31, 107104 (2019); https://doi.org/10.1063/1.5121312

Effects of bulk viscosity on compressible homogeneous turbulence Physics of Fluids 31, 085115 (2019); https://doi.org/10.1063/1.5111062

\section{AIP Author Services English Language Editing}

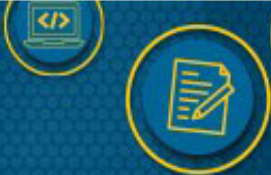




\title{
Probing vortex-shedding at high frequencies in flows past confined microfluidic cylinders using high-speed microscale particle image velocimetry
}

\author{
Cite as: Phys. Fluids 31, 102001 (2019); doi: 10.1063/1.5111817 \\ Submitted: 30 May 2019 Accepted: 9 September 2019 • \\ Published Online: 2 October 2019
}

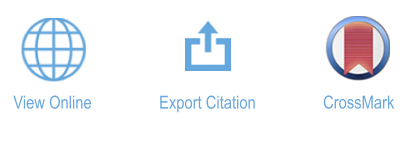

\author{
Shigang Zhang, ${ }^{1}$ (D) Neil Cagney, ${ }^{2, a)}$ (iD Stavroula Balabani, ${ }^{2}$ (D) Carolina P. Naveira-Cotta, \\ and Manish K. Tiwari ${ }^{1,4, a), b)}$ (iD)
}

\begin{abstract}
AFFILIATIONS
${ }^{1}$ Nanoengineered Systems Laboratory, Department of Mechanical Engineering, University College London (UCL), London WClE 7JE, United Kingdom

${ }^{2}$ Department of Mechanical Engineering, University College London (UCL), London WC1E 7JE, United Kingdom

${ }^{3}$ Mechanical Engineering Department (PEM) \& Nanoengineering Department (PENT), Federal University of Rio de Janeiro-UFRJ, Rio de Janeiro, RJ, Brazil

${ }^{4}$ Wellcome/EPSRC Centre for Interventional and Surgical Sciences, University College London (UCL), London, United Kingdom
\end{abstract}

Note: This paper is part of the Special Issue from the 2018 Micro and Nano Flows Conference.

a) Current address: School of Engineering and Materials Science, Queen Mary University of London, United Kingdom.

b) Author to whom correspondence should be addressed: m.tiwari@ucl.ac.uk. Phone: +44 2031081056.

\begin{abstract}
Vortex-shedding from micropins has the potential to significantly enhance and intensify scalar transport in microchannels, for example by improving species mixing. However, the onset of vortex-shedding and the mixing efficiency are highly sensitive to the confinement imposed by the microchannel walls. In this work, the time dependent flow past a cylindrical pin in microchannels with different levels of confinement was studied experimentally. The onset of vortex-shedding in such flows is associated with high, kilohertz range frequencies that are difficult to resolve using conventional laser-based microscale particle image velocimetry ( $\mu$ PIV) techniques. Hence, in this study, a high-speed $\mu$ PIV technique was implemented in order to obtain time-resolved measurements of the velocity fields downstream of the micropin to estimate the corresponding vortex-shedding frequencies and quantify the mixing in the pin wake. The vertical confinement (pin length to diameter ratio) was found to delay the onset of vortex-shedding. When vortex-shedding was present, the shedding frequency and the corresponding Strouhal numbers were found to be greater in channels with higher lateral confinement for the same Reynolds number. Finite-time Lyapunov exponent analysis was performed on the acquired velocity fields to estimate the mixing performance. The results clearly illustrated the significant enhancement in both the mixing in the wake and the mass flux across the centerline of the wake induced by vortex-shedding.
\end{abstract}

Published under license by AIP Publishing. https://doi.org/10.1063/1.5111817

\section{INTRODUCTION}

Flow past a cylinder is a canonical problem in fluid mechanics. The flow configuration has recently also attracted progressively greater attention in microscale flows. For example, in the context of passive micromixers, cylindrical pins are often employed to promote chaotic advection and hence mixing, which is inherently challenging as diffusion tends to dominate in such low Reynolds number flows. Vortex-shedding in the wake of these bluff bodies results in periodic changes in the local direction of flow, inducing crossstream flows and reducing the diffusion time. ${ }^{1}$ Vortex-shedding from cylindrical structures embedded within microfluidic channels also has the potential to promote heat and mass transfer: the oscillatory motion in the wake can induce stretching and folding of 
the species streams, ${ }^{2}$ resulting in mixing ${ }^{3}$ as well as heat transfer enhancement. ${ }^{4}$

While vortex-shedding is a well-documented feature of macroscale flows past cylinders, described in detail in many seminal works, ${ }^{5-7}$ its onset is highly dependent on channel and pin geometry. ${ }^{8}$ These parameters may have a significant effect on the flow in microscale devices, where viscous effects tend to dominate and confinement can have a major impact on the device performance. Flows past cylindrical pins in microfluidic channels are bounded both by the side and top walls, i.e., they experience both high blockage (ratio of cylinder diameter to channel width) and a low aspect ratio (cylinder length to diameter ratio). ${ }^{4}$ The terms lateral $\left(w^{*}=W / D\right)$ and vertical confinement $\left(h^{*}=H / D\right)$ can be employed to describe such microconfined cylinder flows, where $W$ is the channel width, $H$ is the channel height, and $D$ is the cylinder diameter (see Fig. 1) such that a decrease in $w^{*}$ or $h^{*}$ corresponds to an increase in lateral or vertical confinement, respectively. Thus, $w^{*}$ is the reciprocal of the blockage ratio and $h^{*}$ the same as the aspect ratio, which are more commonly used in the context of macroscale flows past cylinders.

Given the importance of such confined systems, flow across confined geometries has been studied both computationally as well as experimentally, albeit a majority of works have concentrated on macroscale flows. A number of studies in the literature examining the effect of lateral confinement have been performed using twodimensional (2D) simulations. ${ }^{9-12}$ Broadly, confinement influences both the onset of vortex-shedding as well as the shedding frequency. Sahin and Owens ${ }^{10}$ found that lateral confinement led to an increase in the critical Reynolds number $\left(R e=U_{m} D / v\right.$, where $U_{m}$ is the maximum velocity upstream of the cylinder and $v$ is the kinematic fluid viscosity) for the onset of vortex-shedding as $w^{*}$ was reduced from 10 to 2 (i.e., the flow became more confined) but remained approximately constant at $R e \approx 120$ as $w^{*}$ was reduced further. They also performed a detailed stability analysis and observed other wake modes for $w^{*} \leq 1.56$, including steady asymmetric flow and asymmetric vortex-shedding. Several 2D numerical studies have focused on the effect of lateral confinement on the vortex-shedding frequency, $f_{s}$, which is typically nondimensionalized in the form of the Strouhal number, $S t=f_{s} D / U_{m}$. Griffith et al. ${ }^{9}$ predicted that confinement caused an increase in the shedding frequency, with $S t$ (based on maximum velocity upstream of the cylinder) increasing from $\sim 0.18$ to $\sim 0.34$ as the confinement was increased from $w^{*}=10$ to

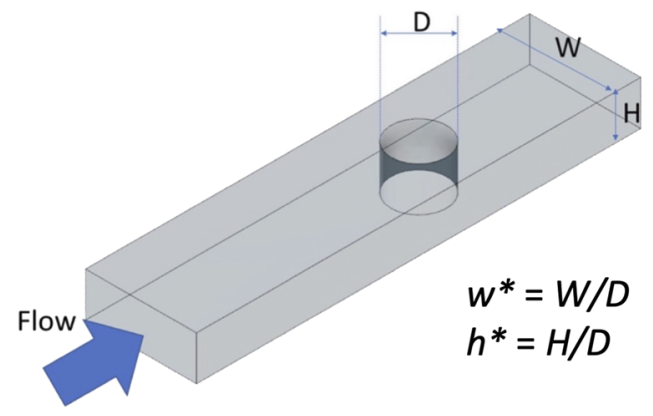

FIG. 1. Schematic of flow past a confined microfluidic cylinder defining the lateral $\left(w^{*}\right)$ and vertical confinement $\left(h^{*}\right)$ parameters. $w^{*}=0.11$ (for $R e=150$ ). A similar trend was observed by Singha and Sinhamahapatra, ${ }^{11}$ who predicted an increase of $S t \approx 0.17$ at $w^{*}=8$ to $S t \approx 0.34$ at $w^{*}=2$ (also for $R e=250$ ). In contrast, Turki et al. ${ }^{12}$ examined a square cylinder in confined flow and observed the opposite trend, in which lateral confinement led to a decrease in $S t$.

Many studies have examined the effect of the aspect ratio or vertical confinement, in flows in which lateral confinement is negligible. $^{13-16}$ The vertical confinement caused a decrease in St, in contrast to the lateral confinement. ${ }^{13,15}$ Inoue and Sakuragi ${ }^{14}$ performed a series of experiments and 3D simulations and found that increasing vertical confinement from $h^{*}=5.5$ to 0.5 led to an increase in St from $\sim 0.2$ to $\sim 0.3$ and a corresponding increase in the critical Reynolds number from $\sim 100$ to $\sim 550$.

In many practical applications such as microscale devices, the values of $w^{*}$ and $h^{*}$ are both small, which may introduce significant three dimensional effects. Various studies have examined the three-dimensional flows past cylinders confined both laterally and vertically. For example, Rehimi et al. ${ }^{17}$ experimentally investigated the flow past a confined cylinder with $w^{*}=3$ and $h^{*}=30$ and found that the critical $R e$ for onset of vortex-shedding increased from 47 to around 108. In a study by Jung et al., ${ }^{18}$ the critical Re reached 400 with $w^{*}=10$ and $h^{*}=1.5$. Reyes et al. ${ }^{19}$ also reported a critical Re value of 340 for a confined channel with $w^{*}=2.5$ and $h^{*}=2.5$ with flow past a square prism. Confinement has also an effect on the length of the separation bubble (recirculation length). Ribeiro et al. ${ }^{8}$ investigated a confined cylinder with $w^{*}=2$ and $h^{*}$ ranging from 16 to 0.5 and showed that for the same $R e$, the recirculation length in the midplane did not change much as the lateral confinement reduced from $h^{*}=16$ to 2 . However, a shorter recirculation length was observed when $h^{*}$ was further reduced to 0.5. The recirculation length was found to vary in the spanwise direction increasing in the proximity of the end walls for the very confined cases $\left(h^{*}=0.5\right.$ and 2$)$.

Despite these efforts, in general, there remain many unresolved questions regarding vortex-shedding in microchannels, such as: how do lateral and vertical confinement influence the wake dynamics and parameters such as the critical Reynolds number and Strouhal number when both $w^{*}$ and $h^{*}$ are small; do secondary shedding modes such as those predicted by Sahin and Owens ${ }^{10}$ occur in real flows; and to what extent are the $2 \mathrm{D}$ and high aspect ratio studies of cylinder flows in the literature relevant to practical microfluidic applications. In addition, there is a lack of experimental data-in particular, time-resolved measurement data- in the literature capturing the unsteady dynamics of vortex-shedding in microscale flows. The aims of this work are two-fold; first, to provide experimental measurements of flow past a cylinder under conditions of extreme confinement in both the lateral and vertical directions, in order to address the outstanding questions discussed above and second, to perform these experiments at the microscale and thus provide the first timeresolved measurements of vortex-shedding in a microfluidic system. The latter step is significant because it demonstrates that it is possible to both manufacture and characterize the performance of micromixers in situ, rather than simply performing macroscale experiments at low $R e$ and similar values of $w^{*}$ and $h^{*}$.

The lack of experimental work on this problem can be attributed to the many challenges this work presents, including the very small time scales involved. This can be appreciated in a very 
straightforward manner as follows. The shedding frequency from a cylinder is given by

$$
f_{s}=\frac{S t U_{m}}{D}=S t \operatorname{Re} \frac{v}{D^{2}} .
$$

Assuming typical values for microfluidic devices with water as working fluid of $S t \sim 10^{-1}, R e \sim 10^{2}, v \sim 10^{-6} \mathrm{~m}^{2} / \mathrm{s}$, and $D \sim 10^{-4} \mathrm{~m}$ a shedding frequency of the order of kilohertz is obtained, which is too high for most microflow measurement systems to resolve. The challenges of using conventional microscale particle image velocimetry $(\mu \mathrm{PIV})$ systems to resolve unsteady flows in micromixers were demonstrated by the study of Renfer et al. ${ }^{20}$ who used a conventional $\mu$ PIV setup to study vortex-shedding dynamics in microcavities with cylindrical micropin arrays. Due to the camera frame rate limitation $(4-10 \mathrm{~Hz})$, the frequency of vortex-shedding could not be determined from the acquired PIV images. Instead, they relied on a high-frequency dynamic pressure sensor; ${ }^{2}$ however, the approach is unable to decipher the detailed flow physics such as velocity maps and vorticity structure across the shear layers, the wake, or the recirculation zone. Given the growing commercial interest in microfluidic devices in general and the vortex-shedding enhanced micromixer, in particular, a time-resolved flow imaging method is required to study such highly dynamic/transient microscale flows. Light Emitting Diode (LED) illumination systems ${ }^{21}$ coupled with a high-speed camera can capture particle images in brightfield mode and overcome these limitations, providing a low cost alternative to measuring fast microscale flows. Brightfield $\mu$ PIV has been demonstrated in applications including microscale blood flows, ${ }^{22}$ flows past swimming zooplankton, ${ }^{23}$ or mixing in a T-Junction ${ }^{24}$ to name but a few. However, to the best of our knowledge, the capability of highspeed brightfield $\mu$ PIV systems to resolve periodic microscale transport phenomena has not been fully demonstrated. Additionally, the fluid dynamics of confined bluff body flows and microscale vortex dynamics also remains unexplored.

In this work, the time-dependent flows past cylindrical micropins are characterized using a custom made high-speed $\mu$ PIV setup. Time-resolved velocity fields are acquired for a range of geometric and flow conditions, and the critical Re, the flow St, wake characteristics, and corresponding vortex-shedding frequencies are determined. The vortex-shedding in channels with vertical confinements $h^{*}$ ranging from 1 to 3 and lateral confinement $w^{*}$ ranging from 2 to 4 are presented. Finally, the Finite-Time Lyapunov Exponent (FTLE) fields are calculated from the measured velocity fields to assess the possibility of scalar mixing in the wake of the micropin.

\section{EXPERIMENTAL SETUP}

\section{A. Microfluidic chip fabrication}

Polymethyl methacrylate (PMMA) rectangular microfluidic channels with a single cylindrical pin (as illustrated in Fig. 1) and different levels of lateral confinement $w^{*}$ were fabricated by computer numerical control (CNC) micromilling (Minitech Machinery, Georgia) and bonded using a thermally solvent assisted bonding technique. ${ }^{25}$ The diameter of the cylindrical micropin was equal to $500 \pm 10 \mu \mathrm{m}$, the channel height ranged from 500 to $1500 \mu \mathrm{m}$ (accurate to within $\pm 30 \mu \mathrm{m}$ ), and the width from 1000 to 2000 (accurate to within $\pm 20 \mu \mathrm{m}$ ), resulting in nondimensional vertical confinements $h^{*}$ ranging from 1 to 3 and lateral ones $w^{*}$ from 2 to 4 . The total channel length was $80 \mathrm{~mm}$, and the distance from the inlet to the micropin was $50 \mathrm{~mm}$ (i.e., $100 \mathrm{D}$ ).

\section{B. Measurement method and experimental procedures}

The experimental setup is shown schematically in Fig. 2. A custom $\mu$ PIV system was assembled comprising a fiber illuminator (150 W, Thorlabs, UK), a microscope assembly (Edmund Optics, UK), and a high-speed camera (Phantom V411, USA). The microfluidic chips were mounted on a three-axis motorized translation stage (Thorlabs, UK). Distilled water was used as the working fluid at room temperature $\left(20^{\circ} \mathrm{C}\right)$. The flow was seeded with $1 \mu \mathrm{m}$ neutrally buoyant polystyrene particles (ThermoFisher, UK) and was driven by a syringe pump (Harvard Bioscience, USA) with flow rates ranging from 0.2 to $105 \mathrm{ml} / \mathrm{min}$ with $\pm 1 \%$ accuracy. The corresponding Reynolds numbers ranged from 2 to 838 (based on the microchannel with the largest cross section area in this study, $w^{*}=3$ and $h^{*}=3$ ). $\mu$ PIV measurements were obtained using brightfield images, with the polystyrene particles acting as tracers. Measurements were taken at various planes along the span of the cylinder with the majority at the midspan. A $5 \times$ microscope objective was used, resulting in a spatial resolution of $4.3 \mu \mathrm{m} /$ pixel. The frame rate ranged between $7.9 \mathrm{kHz}$ and $36 \mathrm{kHz}$ with respective maximum image sizes of $1024 \times 512$ pixels and $320 \times 256$ pixels. In each experiment, 5000 images were acquired and processed using a multi pass cross correlation procedure with a $50 \%$ window overlap, starting with an interrogation window of $128 \times 64$ pixels and ending with $32 \times 32$ pixels.

The present $\mu$ PIV technique was validated against measurements obtained with a laser $\mu$ PIV system (operated at low frame rates) of the flow upstream of the pin for steady flow. The system (not shown here for brevity) comprised a pulsed $16 \mathrm{~Hz} \mathrm{Nd}$-Yag laser (Litron Laser, UK) and a CCD camera (Andor, UK), and otherwise replicated the system detailed above. Figure 3 shows a comparison between the mean axial velocity profiles upstream of the pin, determined using the two techniques. It should be noted that the velocity profile is normalized by the mean velocity upstream of the cylinder $\left(U_{o}\right)$. It can be seen that the two profiles agree within $2 \%$, demonstrating that the high-speed brightfield $\mu$ PIV technique can capture

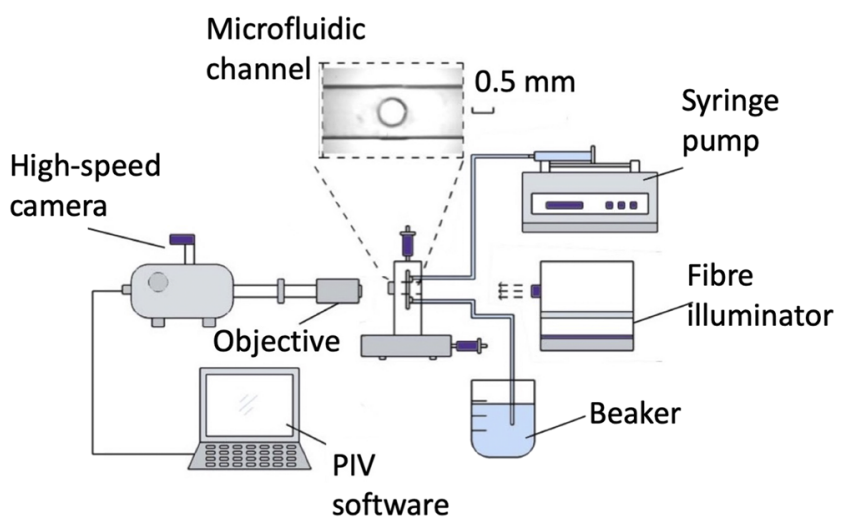

FIG. 2. Schematic of the experimental setup. Inset shows a typical confined microfluidic cylinder $\left(D=500 \mu \mathrm{m}\right.$ diameter and $\left.w^{*}=2\right)$. 


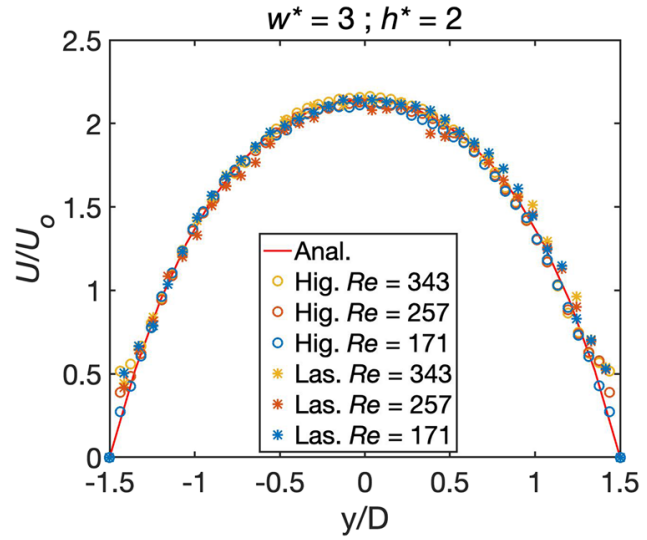

FIG. 3. Mean axial velocity profiles upstream of the micropin $(x / D=-10)$ normalized with the average velocity, $U_{0}$, measured by high-speed brightfield (circle symbols) and double-pulsed Nd:YAG laser $\mu$ PIV (star symbols) at different $R e$. The analytical (solid line) solution is also shown for comparison.

the steady flow field equally well. Normalized velocity profiles such as those shown in Fig. 3 have the advantage that they remove any systematic errors emanating from the depth of correlation. However, it is reasonable to expect errors near the wall to be slightly higher, due to reflections, seeding or large velocity gradients, and this could lead to slightly overestimation of the normalized velocities near walls, as is apparent in Fig. 3 for both $\mu$ PIV approaches used herein. In addition, the uncertainty in the PIV measurements was estimated using the difference between the primary and secondary peak in the cross correlation matrices (see Ref. 26). This was implemented using the PIV software (Insight4G, TSI), and found to be $2 \%$, and is expected to be lower in practice, due to the temporal averaging of the data performed when calculating $U_{m}$. The uncertainty in the pin diameter was $\pm 10 \mu \mathrm{m}$, i.e., $2 \%$. With room temperature remaining between $17^{\circ} \mathrm{C}$ and $20^{\circ} \mathrm{C}$, the resulting viscosity uncertainty is around $4 \%$. From error propagation, we can calculate the error in the Reynolds number to be around $5 \%$.

However, in the current brightfield PIV (imaged using white light) setup, the depth of correlation ${ }^{27}$ can be as high as $\approx 160 \mu \mathrm{m}$, whereas that for a laser based PIV setup $\approx 120 \mu \mathrm{m}$. It is well documented in the literature that the velocities measured using $\mu$ PIV systems, whether brightfield or laser/fluorescence based, are strongly affected by the errors arising from the depth of correlation, ${ }^{28}$ with maximum velocity underestimates reaching a factor of $2 / 3$. Such systematic errors are integral to $\mu$ PIV measurements, and hence, we have not made any attempts to correct and/or scale our measured velocity vectors. Rather, all velocity measurements are normalized by the average velocity determined from the measured profiles, as mentioned above, in order to minimize errors due to the depth of correlation. Flows up to $R e=620$ could be resolved with the current setup.

\section{RESULTS AND DISCUSSION}

\section{A. Upstream flow conditions}

In order to verify the upstream flow conditions, velocity measurements were taken at different spanwise planes and compared

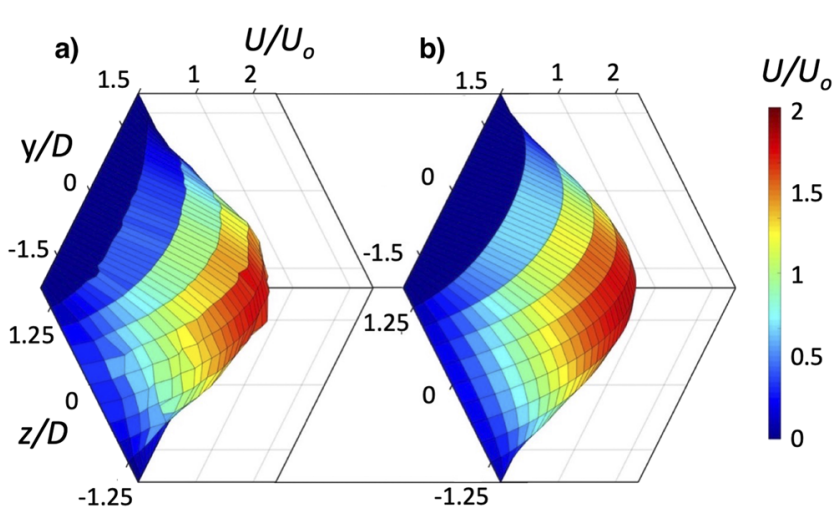

FIG. 4. (a) Three dimensional velocity measurements of the flow approaching the micropin and (b) the analytical solution in a microchannel with $w^{*}=3$ and $h^{*}=2.5$ at $R e=151$. Velocities are normalized with the average velocity obtained from the PIV measurements.

with the 3D analytical solution for fully developed flow in a rectangular channel. ${ }^{29}$ The origin of the coordinate system is located at the axis of the pin and at midspan. The $x, y$, and $z$ axes denote the streamwise (flow), transverse, and spanwise directions, respectively. The velocity profile for laminar, fully developed flow in a rectangular channel can be written as

$$
U_{x}(y, z)=\frac{4 H^{2} \Delta p}{\pi^{3} \eta L} \sum_{n, o d d}^{\infty} \frac{1}{n^{3}}\left[1-\frac{\cosh \left(n \pi \frac{y}{H}\right)}{\cosh \left(n \pi \frac{w}{2 H}\right)}\right] \sin \left(n \pi \frac{z}{H}\right),
$$

where $\Delta p$ is the pressure drop along the channel and $\eta$ is the dynamic viscosity. Figure 4 shows a comparison between the velocity measurements and the analytical solution in a microchannel with $w^{*}=3$ and $h^{*}=2.5$ at $R e=151$. The experimental data were acquired at $x / D=-10$ (i.e., $10 D$ upstream the center of cylindrical pin) and at different $\mathrm{z}$ planes from -1.25 to $1.25 \mathrm{D}$ and were combined to obtain the $3 \mathrm{D}$ flow velocity upstream of the micropin. The velocity profiles were normalized by the average velocity calculated from the PIV measurements.

In order to make the comparison more quantitative, selected velocity profiles measured at the channel midplane $(z / D=0)$ for different confinements and $R e$ are compared against the analytical solution in Fig. 5. Excellent agreement with the analytical solution (within 1\%) can be observed, providing confidence that the flow approaching the pin is fully developed and reproducible. The curvature in the velocity profile upstream plays a key role in the development of the flow downstream of the cylinder and is affected by the vertical confinement, as Fig. 5 demonstrates.

\section{B. Velocity fields and onset of instability}

In order to investigate the role of vertical confinement on the wake dynamics and mass transfer, a series of measurements were performed in which the lateral confinement was held constant at $w^{*}=3$ and the vertical confinement was varied between $h^{*}=1$ and 3. The supplementary material shows an example of the kind of velocity maps and vorticity maps obtained for all the geometries studied. Sample instantaneous vorticity fields, $\omega_{z}$, are shown in Fig. 6 for a range of Reynolds numbers and three different values of $h^{*}$. 

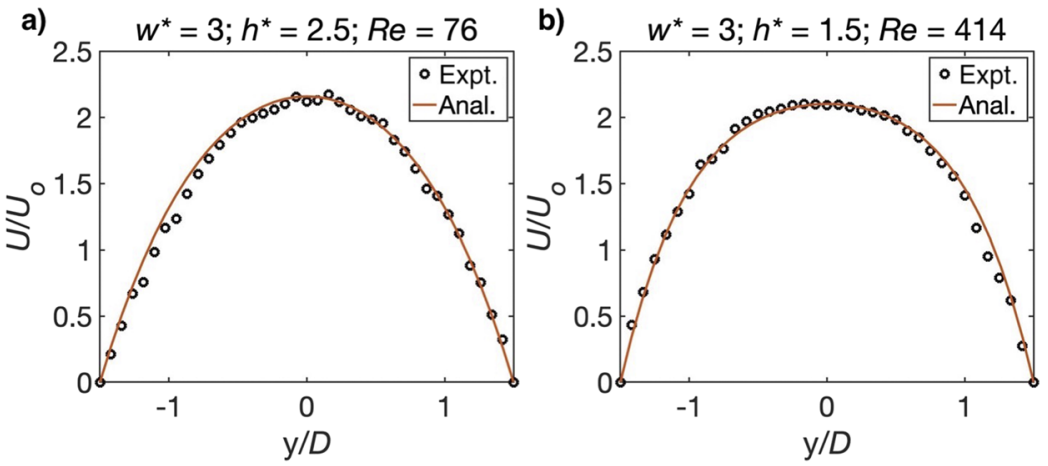

FIG. 5. Comparisons between experimental (symbols) and analytical (solid line) velocity profiles upstream of the micropin $(x / D=-10, z / D=0)$ for two microchannels with $w^{*}=3$ and (a) $h^{*}=2.5$ and (b) $h^{*}=1.5$, respectively.

In all cases, at low $R e$, the wake is characterized by two long shear layers which form at the lateral edges of the cylinder and extend downstream but do not roll up to form vortices (Fig. 6, top row). Downstream of the cylinder, the shear layers gradually move close together and converge upon the wake centerline $(y=0)$. The roll-up of the shear layers and formation of vortices can be seen in $h^{*}=2$, $R e=448$ and $h^{*}=3, R e=304$, respectively. In contrast, for the case with the strongest vertical confinement $\left(h^{*}=1\right)$, roll-up is not present in any of the vorticity fields in Fig. 6, and the structure of the vorticity fields remains symmetrical. This indicates that vertical confinement acts to increase the critical Reynolds number for the onset of vortex-shedding and highlights the significant stabilizing effect of the end walls.

Similar to the previous figure, Fig. 7 presents instantaneous vorticity and velocity fields for three cases in which the vertical confinement is held constant at $h^{*}=2$ and the Reynolds number and lateral confinement are varied. Shear layers along the channel walls are apparent in all fields in Fig. 7; however, for $w^{*}=2$, the confinement is so great that these shear layers appear to be in contact with the ones forming at the cylinder, and both sets of shear layers have comparable vorticity magnitude. The strength of the vorticity in the shear layers is greatest in the most confined case, due to the increased
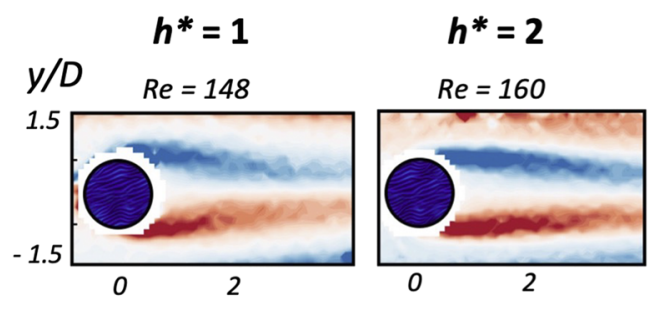

$$
h^{*}=\mathbf{3}
$$
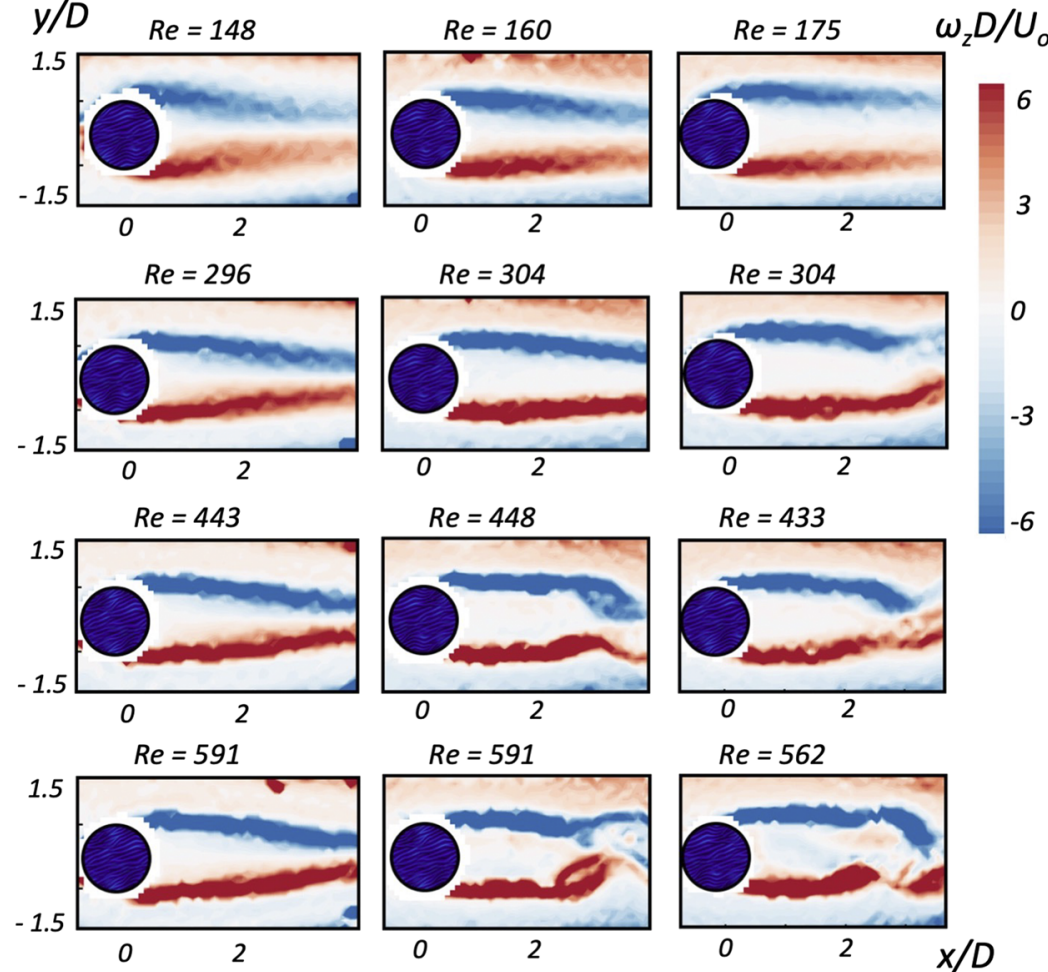

FIG. 6. Instantaneous normalized vorticity contours $\omega_{z} D / U_{0}$ for a channel with $w^{*}=3$ and $h^{*}$ ranging from 1 to 3 , for different $R e$. 

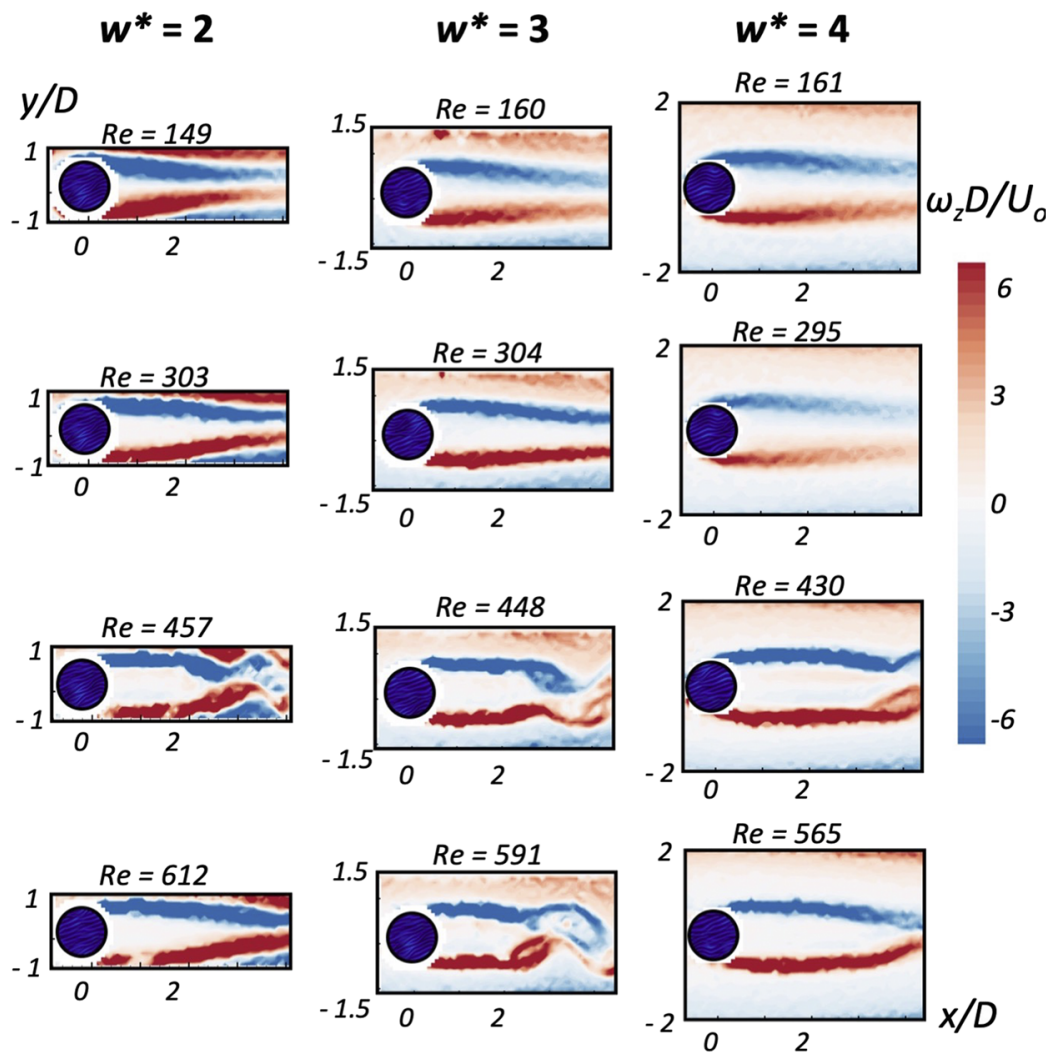

FIG. 7. Instantaneous normalized vorticity contours for a channel with $h^{*}=2$ and $w^{*}$ ranging from 2 to 4 , for different Re.

velocity of the fluid between the cylinder and the side walls. Conservation of mass shows that the mean velocity of the fluid in the gap between the cylinder and the wall is given by

$$
U_{\text {gap }}=U_{o}\left(\frac{w^{*}}{w^{*}-1}\right)
$$

where $U_{o}$ is the mean velocity upstream of the cylinder. For $w^{*}=2$, this leads to a doubling of the mean streamwise velocity, which explains the strength of the vorticity seen in the left column in Fig. 7. In this case of strong lateral confinement, we can observe two sets of shear layers. One is near the channel wall (i.e., wall boundary layer) and the other, the free shear layer resulting from the separation of the symmetrically formed boundary layers that originate from the cylinder. On either side of lateral symmetry, between these two set of shear layers, we observe a region of relatively stronger streamwise, jetlike flow (due to flow continuity); this is particularly the case for cases with strong confinement (i.e., $w^{*}=2$ ). Interestingly, in the case of strongest confinement in Fig. 7 (i.e., $w^{*}=2$ ), we observe two sets of vortices on either side of the lateral symmetry plane. First, the roll-up of free shear layers is observed near $x / D \approx 2.5$ for $R e=457$ (see the negative vorticity, blue vortex). This is also followed by formation of an oppositely signed (red) vortex near the channel wall, slightly downstream (at $x / D \approx 3$ ). For $w^{*} \geq 3$, the shear layers at the wall and the cylinder are separated by a region of weak vorticity and vortex formation does not coincide with noticeable changes in the vorticity at the walls.
Due to the significant vertical confinement $\left(h^{*}=2\right)$, no vortexshedding is observed for Re up to 280 in all $w^{*}$ confined cases. Shear layer roll up and vortex formation becomes apparent for $R e>440$ in all microchannels. The lateral confinement appears to have a weaker effect on the critical $R e$ for the onset of flow instability and vortexshedding in the range $\left(w^{*}=2-4\right)$ studied here, compared to that of vertical confinement (Fig. 6). In all the sets of PIV measurements examined, the flow was characterized either by a steady, symmetric wake (below the critical Re) or alternate shedding, as shown in Figs. 6 and 7. At no point did we observe any of the other flow regimes discussed by Sahin and Owens ${ }^{10}$ (based on 2D simulations) for slightly greater confinement $\left(w^{*} \leq 1.56\right)$, which may be a result of larger $w^{*}$ values examined in the present experiments.

The shedding of alternate vortices from the micropins breaks the symmetry of the flow and induces a transverse velocity component along the centerline of the wake. Hence, the distribution of the transverse velocity component is a suitable means to identify the critical Reynolds number for the onset of instability. Selected contours of the instantaneous transverse velocity for the case with $w^{*}=3$ and $h^{*}=2$ at two different Re numbers 304 (prior to the onset of vortex-shedding) and 448 (with vortex-shedding) are shown in Fig. 8. Prior to vortex-shedding, low (almost zero) transverse velocities are observed in the wake of the micropin, especially along the wake centerline $(y / D=0)$. However, once vortex-shedding is present, significant crossflow across the centerline is evident from the increased transverse velocity values in the wake. The variation of the maximum (absolute) value of the transverse velocity along the 


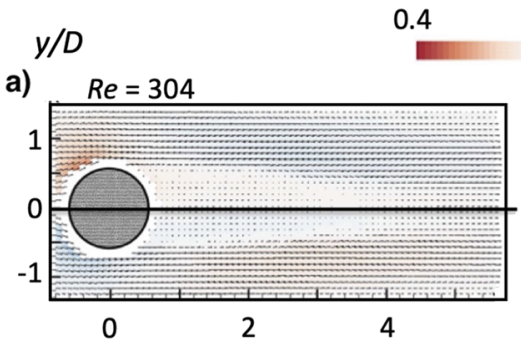

\section{$0 \quad-0.4 V(\mathrm{~m} / \mathrm{s})$}

b) $\quad R e=448$

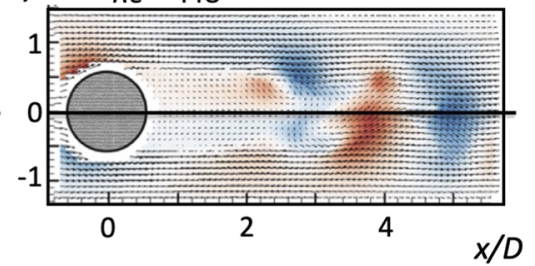

FIG. 8. Contours of instantaneous transverse velocity $V$ with velocity vectors superimposed for a channel with $h^{*}=2$ and $w^{*}=3$ and for (a) $R e=304$ (no vortex-shedding) (b) $R e=448$ (with vortex-shedding). wake centerline, $\left|V_{\max }\right|$, with $R e$ and confinement, is shown in Fig. 9. It can be seen that $\left|V_{\text {max }}\right|$ is approximately zero for the low $R e$ range and increases sharply beyond a critical Re value, which marks the onset of vortex-shedding.

The critical value of Re is thus identified from Fig. 9 and plotted as a function of confinement in Fig. 10. The present results illustrate an almost monotonic increase of the critical Re from 220 to 550 with vertical confinement $h^{*}$ [Fig. 10(a)]. The critical Re remains significantly larger than the values known for unbounded cylinders. ${ }^{3}$ In contrast, the variation of critical Re with lateral confinement for the $\mathrm{h}^{*}=2$ case is not as pronounced; the onset of vortex-shedding occurs within a relatively narrow range of $\operatorname{Re} \approx 280-350$ and a slight reduction of the critical $R e$ with increasing lateral confinement can be discerned [Fig. 10(b)]. This is possibly due to an increase in the maximum streamwise velocity past the cylinder with decreasing $w^{*}$ (i.e., there is an increase in the velocity in the gap between the cylinder and the lateral wall). In Fig. 10, we also show results from macroscale cylinder flow literature, though strictly the latter are not for cases with confinement in both directions. Similarities and differences are clearly apparent. First, in Fig. 10(a), the increase of vertical confinement (decreasing $h^{*}$ ) even at large values of $w^{*}$ (negligible lateral confinement) shows a trend similar to our results. The literature results are from the computation study by Inoue and Sakuragi ${ }^{14}$ and the experiments of Jung et al. ${ }^{18}$ who only investigated one vertical confinement. In Fig. 10(b), the results from the literature at high lateral confinement (i.e., $1.3<w^{*}<5$ ) but relatively low or no vertical confinement $\left(h^{*}=30\right)$ show the critical Reynolds number to be around 100. The literature results in Fig. 10(b) are from the simulations of Sahin and Owens ${ }^{10}$ and the experiments by Rehimi et $a l .{ }^{17}$ Our results (in black), show the critical Reynolds number to be substantially higher $(\sim 350)$; clearly, the vertical confinement has a more pronounced effect. However, the relative invariance of the critical Re with $w^{*}$ is maintained. For completeness, we also note the macroscale investigation by Shair et al., ${ }^{31}$ who studied the effect of lateral confinement in the range of $20-5$, and found that the critical Reynolds increases with increasing lateral confinement (decreasing $w^{*}$ ) because the wake instability is inhibited by the presence of the walls. However, Sahin and Owens ${ }^{10}$ reported an opposite
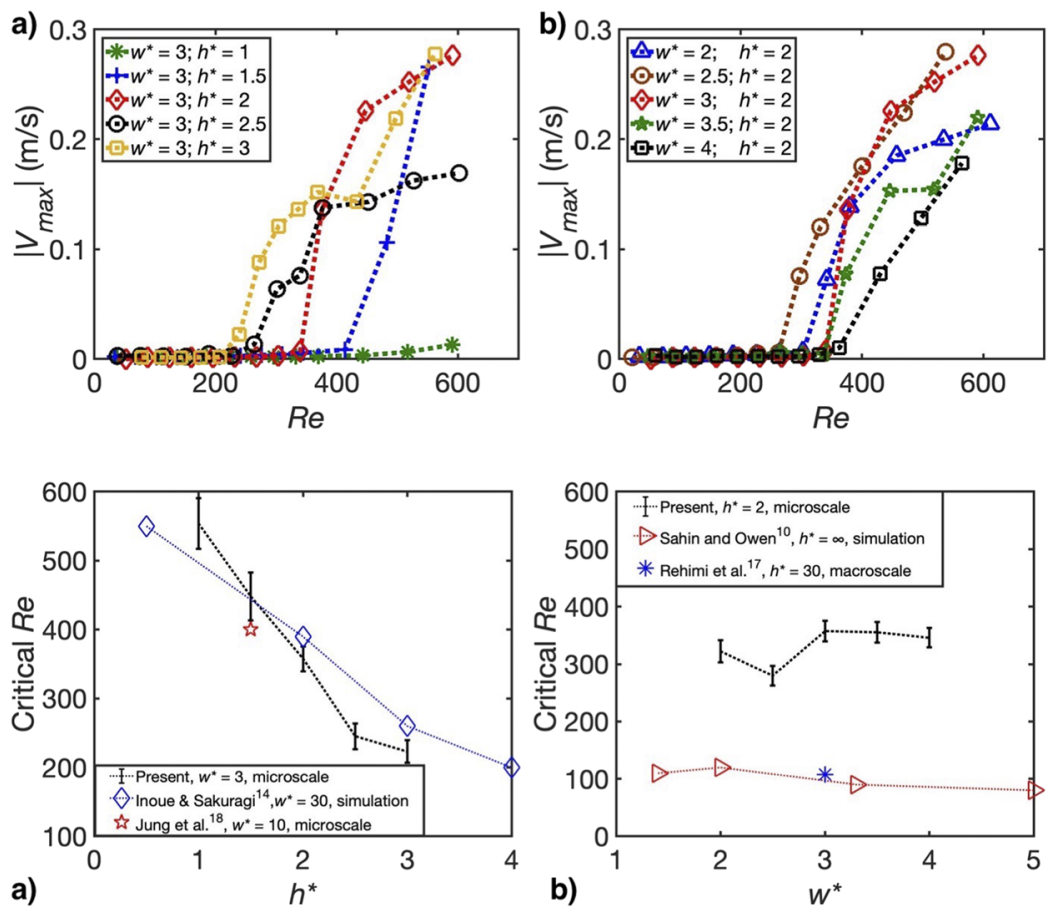

FIG. 9. Variation of the maximum transverse velocity along the wake centerline $(y / D=0)$ with $R e$, for various values of (a) vertical and (b) lateral confinement.
FIG. 10. The relationship between critical $R e$ and (a) vertical and (b) lateral confinement. Comparative results from literature are also included. The error bars in our data (in black symbols) represent the measuring gap between the $R e$, i.e., the difference in $R e$ between successive sets of $\mu$ PIV measurements. 
$y / D \quad R e=88$

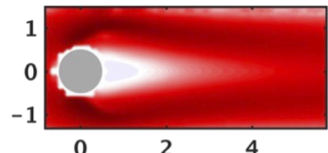

0

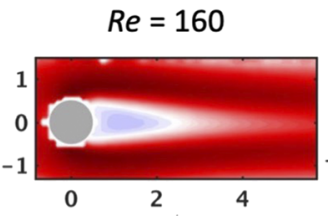

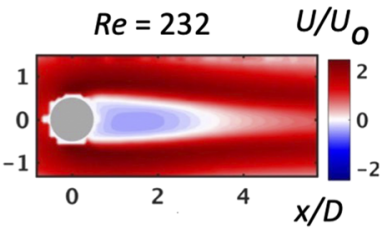

FIG. 11. Contours of the normalized axial mean velocity for a channel with $h^{*}=2$ and $w^{*}=3$ and for different $R e$. trend at $w^{*}<2$, finding a modest rise in critical Re due to an increase in the fluid velocity in the gap between the cylinder and the wall with increase in the lateral confinement (decreasing $w^{*}$ ). However, these changes are not as pronounced as the vertical confinement, resulting in a relatively higher critical $R e$ for all our investigated lateral confinement cases with $h^{*}$ fixed at 2 . At the same Reynolds number, the change in vertical confinement does not alter the mean gap velocity. Therefore, we only observe the proximity of vertical walls leading to a substantial stabilization of the wake instabilities and, consequently, an increase of the critical Reynolds number.

Overall, the results indicate that the lateral confinement in microfluidic devices suppresses vortex-shedding from micropins, delaying the transition to the unsteady wake regime, in agreement with the predictions of various $2 \mathrm{D}$ simulations. ${ }^{9-11}$ The data show that this occurs also in three-dimensional flow and in the presence of significant vertical confinement. However, our results indicate that the vertical confinement (i.e., reduction in $h^{*}$ ) has a much greater effect on the critical Reynolds number than the lateral onesuggesting that $2 \mathrm{D}$ studies in which vertical confinement is neglected may have limited relevance to practical microfluidic devices.

\section{Recirculation length}

Prior to the onset of vortex-shedding, a stationary vortex pair forms behind the pin and remains attached to the structure, forming a recirculation region. ${ }^{32}$ Figure 11 shows axial velocity contours where red indicates positive values (i.e., flow directed away from the pin-downstream), blue the negative values (i.e., flow reversal), and white regions with zero velocity mark the recirculation region downstream of the pin. The recirculation length, $L$, was taken as the distance between the center of the micropin and the point along the centerline where the mean streamwise velocity was zero, $U=0$. Figure 12 shows the variation in the recirculation length as a function of Reynolds number for various $w^{*}$ and $h^{*}$, for the cases prior to the onset of vortex-shedding. The length of the recirculation region generally increases with $R e$ for the range investigated in agreement with published studies. ${ }^{8}$ The recirculation length shows an almost monotonic dependence on lateral confinement [Fig. 12(b)], whereas the relationship with $L / D$ on $h^{*}$ is not strictly monotonic. This is in agreement with the numerical study of Singha and Sinhamahapatra ${ }^{11}$ and Ribeiro et al.

\section{Vortex-shedding frequency and Strouhal number}

The shedding frequency is one of the most important properties of time-dependent flows past bluff bodies. Fast Fourier transform (FFT) was applied to the instantaneous transverse velocity signal at a selected point downstream of the micropin $(x / D=4, y / D$ $=1$ ) to determine the shedding frequency. Figure 13(a) shows a typical transverse velocity signal in the wake of the pin extracted from a)

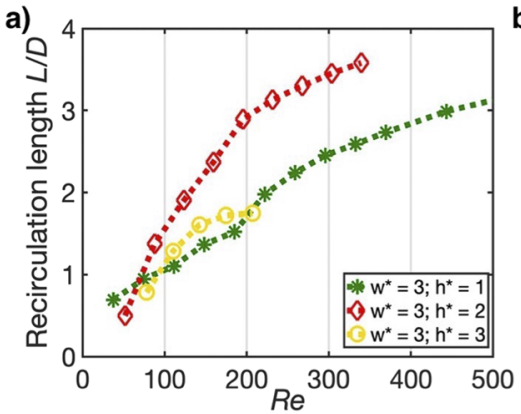

a)

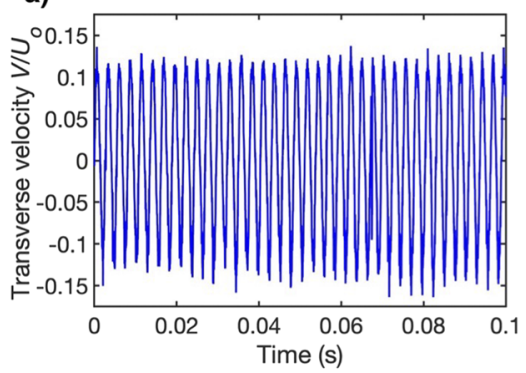

b)

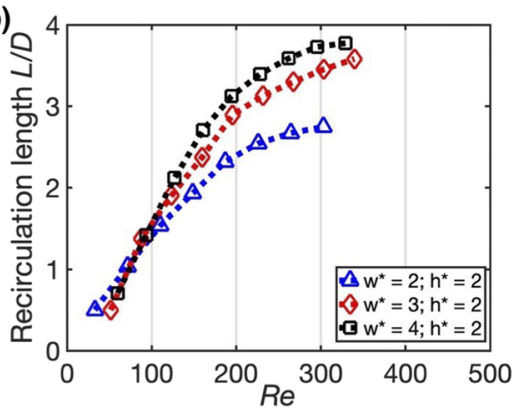

b)

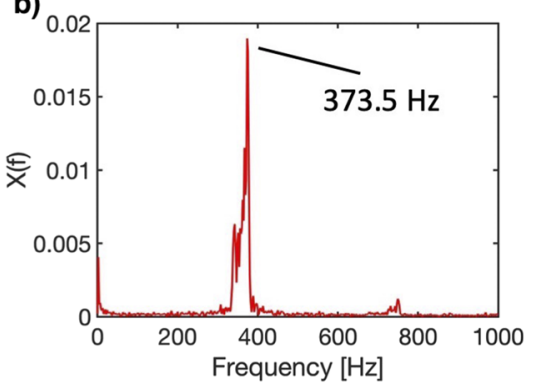

FIG. 12. Length of the recirculation region for different $R e$ and various values of (a) vertical confinement and (b) lateral confinement.
FIG. 13. (a) Instantaneous normalized transverse velocity $\left(V / U_{0}\right)$ signal at a selected point downstream of the pin in a channel with $w^{*}=3$ and $h^{*}=2$ at $R e=340$ and (b) the corresponding amplitude spectrum showing a distinct vortex-shedding peak. 


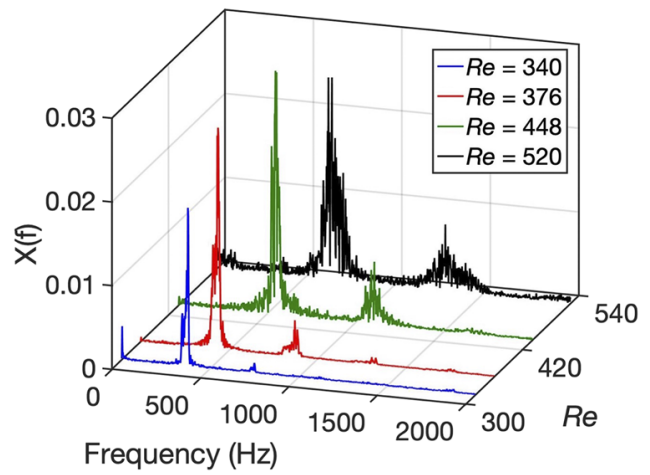

FIG. 14. Frequency spectra of the $V / U_{0}$ signals at different $R e$ for a channel with $w^{*}=3$ and $h^{*}=2$. The second peaks in each case shown are harmonics.

an instantaneous flow field (obtained at a frame rate of $36 \mathrm{kHz}$ ) for a channel with $w^{*}=3$ and $h^{*}=2$ at $R e=340$. A dominant frequency peak at $373.5 \mathrm{~Hz}$ can be detected from the FFT spectra [Fig. 13(b)] corresponding to the frequency of vortex-shedding. In order to obtain more reliable results, two additional points at the same downstream location $(x / D=4)$ but different lateral positions $(y / D=0$ and 1$)$ were examined in each experiment and an average value of the vortex-shedding frequency was taken. The maximum standard deviation in the measured frequency was $4.9 \%$ of the mean. Amplitude spectra for different $R e$ and a channel with $w^{*}=3$ and $h^{*}=2$ are shown in Fig. 14, illustrating that the vortex-shedding frequency increases with $R e$ for the same channel as expected. The measured vortex-shedding frequencies are very high, well above the maximum resolvable range of conventional $\mu$ PIV systems, highlighting the value of the high-speed imaging based approach followed here.

The relationship between the shedding frequency and Re for different confinements is shown in Fig. 15. The St-Re data are plotted both in terms of the maximum upstream velocity [Figs. 15(a) and 15(b) panel] and the maximum gap velocity [Eq. (3)], i.e., $S t_{\text {gap }}$ $=f_{s} D / U_{\text {gapm }}, R e=U_{\text {gapm }} D / v$ [Figs. 15(c) and 15(d)]. The blue dottedline [in Figs. 15(a) and 15(b)] indicates the St-Re relationship for unconfined flow based on the studies of Fey et al. ${ }^{33}$ and is used as a reference. In the range of confinements and $R e$ investigated herein, Figs. 15(a) and 15(c) show that St is virtually independent of $h^{*}$ and increases monotonically with Re. However, for lower values of $h^{*}$ ( 1 and 0.5 ), no dominant frequency was detected from the spectra of the fluctuating velocity implying that the onset of vortex-shedding is further delayed to Re higher than those for the cases shown in Figs. 15(a) and 15(c). We note that higher Re was inaccessible using the syringe pump system used in our work.

Visual inspection of the velocity vectors and vorticity contours such as those shown in Figs. 6 and 7 indicates that vortices started shedding when $R e$ reached 550 or 450 , for $h^{*}$ values 1.5 and 1 , respectively. This illustrates the significant retardation effects of the end walls as discussed in Sec. III B. Figures 15(c) and 15(d) present the relationship between the Strouhal number and Reynolds number based on the maximum gap velocity measured with the PIV system, $U_{\text {gapm }}$, which is also commonly used in the studies of vortex-shedding in confined cylinder pins. ${ }^{34,3}$

On the contrary to the vertical confinement, lateral confinement $w^{*}$ seems to increase St values [see Fig. 15(b)]. The St values appear similar for the most confined cases, whereas those for the less confined case appear to become independent of $R e$ and
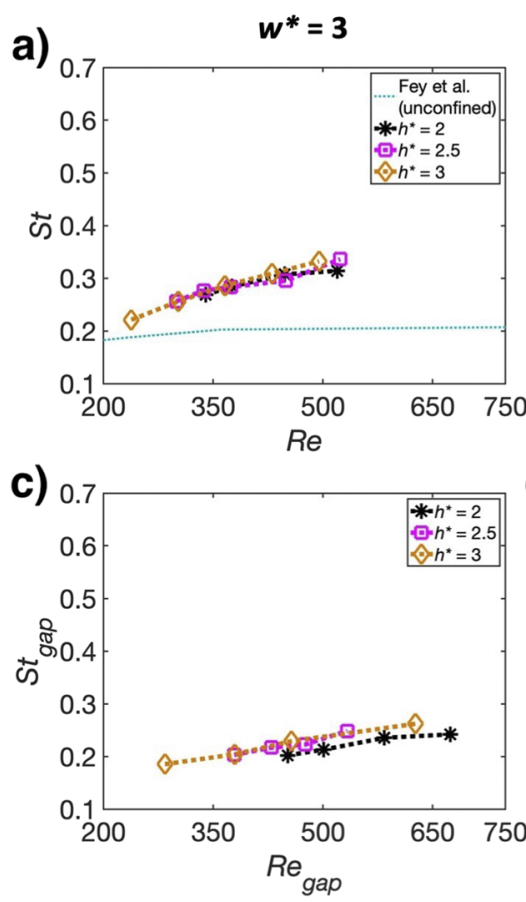
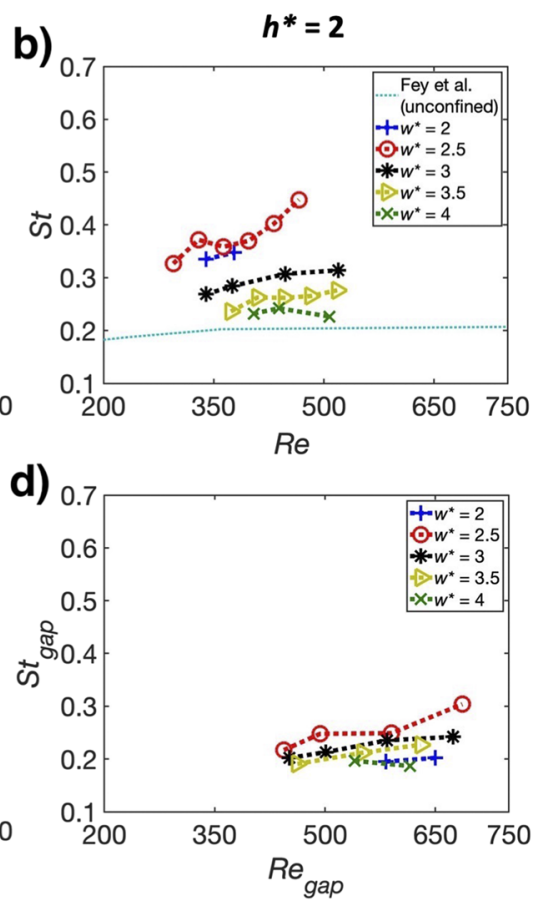

FIG. 15. The relationship between $S t$ and $R e$ for various values of [(a) and (c)] vertical confinement and [(b) and (d)] lateral confinement. The dashed line represents the measured values in the case of negligible confinement. ${ }^{3}$ (c) and (d) are calculated based on maximum gap velocity. 
even approach the $S t$ for the unbounded case when $w^{*}=4$. The increase of St with lateral confinement (decreasing $w^{*}$ ) is in agreement with the findings of Griffith et al. ${ }^{9}$ and Alfieri et al., ${ }^{36}$ which can be understood by the increase of the mean gap velocity with the wall proximity in lateral confinement. The strength of the vorticity in the shear layers becomes greater in cases with strong lateral confinement, due to the increased velocity of the fluid between the cylinder and the walls, resulting in a higher shedding frequency and St.

Figure 15 essentially captures two main aspects of the confined vortex-shedding phenomenon: the relative independence of $S t$ on vertical confinement and the gap velocity playing an important role in controlling the shedding frequency and St. The gap velocity is independent of $h^{*}$ [Eq. (3)] but depends strongly on $w^{*}$ when $w^{*}$ is small (significant lateral confinement). This explains why the Strouhal number is independent of $h^{*}$ but varies with $w^{*}$. In Fig. 15, using the maximum gap velocity, captured from the $\mu$ PIV measurements, and replotting Fig. 15(b) as Fig. 15(d) brings St data closer together and results in St values around 0.2.

The exact way in which $w^{*}$ affects the gap flow (e.g., the location and magnitude of the maximum of the gap flow) will depend strongly on the state of the boundary layer both around the cylinder and along the channel walls, which in turn will depend strongly on the Reynolds number. This is likely to be the cause of the $R e$-dependence of the Strouhal number.

\section{E. Lagrangian analysis}

In order to analyze the effect of vortex-shedding on microscale transport in the wake, we studied the Lagrangian characteristics of the flows measured. This was achieved by numerically advecting tracers through the phase-average velocity fields in order to identify where individual regions of fluid will move to or have originated from. A commonly used Lagrangian metric of mixing is the
Finite-Time Lyapunov Exponent (FTLE), which is a measure of the rate of fluid stretching. ${ }^{3}$

In order to calculate the FTLE fields, it is first necessary to find a flow map, $\varphi$, which represents the positions of a series of massless "particles." By tracking the rate at which neighboring particles separate with time, the rate of stretching can be measured. In practice, this is found by assuming initial positions, $\boldsymbol{x}_{0}=\left(x_{0}, y_{0}\right)$, of the particles at some time $t_{0}$ and evaluating how they vary with time by integrating the velocity field,

$$
\frac{d}{d t}\left(\varphi\left(\boldsymbol{x}, t, t_{o}\right)\right)=\mathbf{u}\left(\boldsymbol{x}_{\boldsymbol{o}}, \mathrm{t}, t_{o}\right)
$$

where $\boldsymbol{u}=(U, V)$. The rate of exponential stretching by neighboring particles in chaotic flow that tend to separate exponentially is given by

$$
\sigma_{f}=\frac{1}{\left|t-t_{o}\right|} \log \sqrt{\lambda_{\max }}
$$

where $\lambda_{\max }$ is the largest eigenvalue of the matrix $(\nabla \varphi)^{T}(\nabla \varphi)$.

Regions of high $\sigma_{f}$ indicate regions of fluid that will undergo rapid stretching. The positions of tracers can also be found by integrating the tracers backward in time (i.e., by reversing the direction of flow), in which case regions of high $\sigma_{\mathrm{f}}$ correspond to points where the fluid has recently undergone intense stretching. The superscript "+" and "-" are used to denote whether the FTLE fields were computed using forward or backward integration. The FTLE fields are an effective means of identifying coherent structures within a flow and characterizing the regions where mixing is most efficient. ${ }^{37}$ Cagney and Balabani ${ }^{3}$ used this approach and demonstrated that various modes of vortex-shedding from an oscillating cylinder can induce different levels of mixing.

The normalized FTLE fields computed in forward- and backward-time are shown in Fig. 16 for a range of $R e$, for $w^{*}=3$,
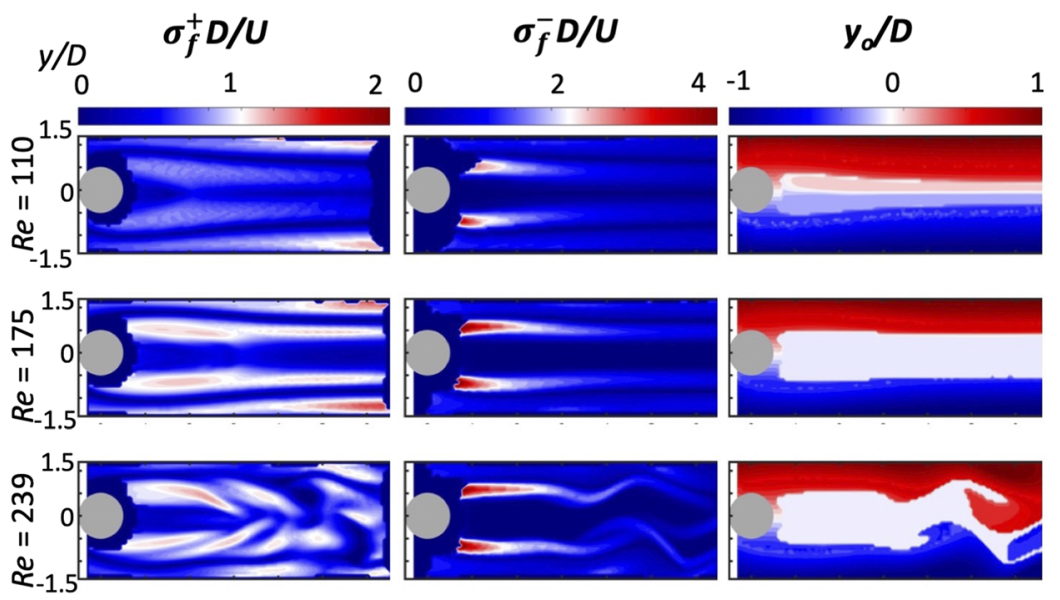

FIG. 16. FTLE fields for a microchannel with $h^{*}=3$ and $w^{*}=3$ at different Re; forward-time FTLE (left column), backward-time FTLE (middle), and tracer fields (right). The tracer fields indicate the transverse position from which each region of the fluid originated from. All data is shown for a single phase in the shedding cycle.
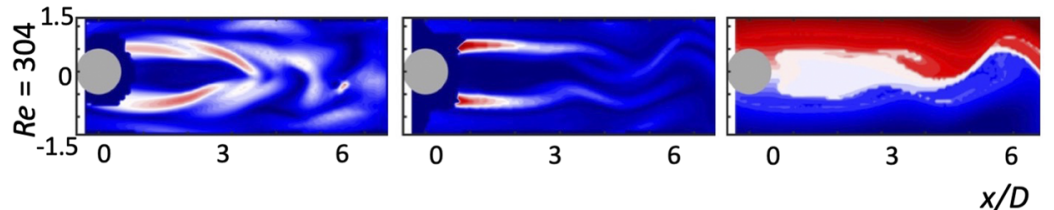
$h^{*}=3$. It can be seen that before the onset of vortex-shedding $(R e=110$ and 175), the regions of rapid stretching in forwardand backward-time occur primarily at the channel walls and in narrow bands at $y / D \approx 0.75$, which approximately corresponds to the boundary between the recirculation region and the rest of the flow. However, once vortex-shedding occurs, regions of intense stretching are distributed more evenly throughout the flow, as can be seen in the lower two rows of Fig. 16. This indicates that the vortexshedding enhances mixing in the wake of the pin. The right column in Fig. 16 indicates the transverse origin, $y_{o}$, of tracers throughout the wake at a single phase. These "tracer fields" are found by advecting the fluid tracers backward in time over five shedding cycles (or the equivalent time in the cases without vortex-shedding) and identifying the lateral position at which they entered the flow domain. This is particularly relevant if the intention of the pin is to enhance scalar (species concentration or thermal energy) transport across the channel, and the aim is therefore to enhance mass flux from one side of the channel to the other. It can be seen that at low Re, before the onset of vortex-shedding, there is negligible cross-wake mixing, i.e., fluid tracers on the upper side of the channel $(y / D>0)$ all originate on the upper side of the cylinder, $\left(y_{o} / D>0\right.$ also $)$, and likewise for $(y / D<0)$. However, at higher $R e$, when vortex-shedding is present, a significant proportion of the fluid near the center of the wake originated close to the channel walls.

It can clearly be seen that when $R e$ exceeds the critical value for the onset of vortex-shedding, a significant increase in cross-wake mixing occurs, illustrating the ability of our approach to assess the improvement in mixing in microchannels due to the insertion of micropins. Based on the correlation between mass transfer and heat transfer, ${ }^{38,39}$ the improvement of mixing also indicates heat transfer enhancement.

\section{CONCLUSIONS}

In this work, a new experimental system was developed to fully resolve microscale time-dependent flows. The system made use of high speed $\mu$ PIV measurements in brightfield mode in order to explore the role of microchannel confinement on the onset of vortexshedding from a single cylindrical micropin, with a view to enhancing scalar transport. The vertical confinement was found to strongly suppress/delay vortex-shedding with the critical $R e$ for the onset of shedding increasing from 220 to 550 when $h^{*}$ reduced from 3 to 1 (in channels with $w^{*}=3$ ). Lateral confinement on the other hand did not alter the critical Reynolds number too significantly; it remained between 280 and 350 in the tested range of $w^{*}=2-4$, for a fixed $h^{*}=2$. However, lateral confinement was found to have a stronger effect on the shedding frequency than vertical confinement, with $S t$ increasing with a reduction of $w^{*}$ at the same $R e$. The reduction of $w^{*}$ increased the velocity of the fluid between the cylinder and the walls, resulting in greater strength of the vorticity in the shear layers. Lagrangian analysis of the measured flow fields clearly showed that vortex-shedding enhances the mixing performance and material transport due to the generation of a significant crossflow in the wake of the pin.

\section{SUPPLEMENTARY MATERIAL}

See supplementary material for FFT spectra found using the transverse velocity signals extracted from the PIV measurements at various points outside and inside the recirculation zone for $\operatorname{Re}=340$, $w^{*}=3, h^{*}=2$. Supplementary Video: The time-resolved measurement of high-speed vortex-shedding from confined micropin with $w^{*}=3, h^{*}=2$.

\section{ACKNOWLEDGMENTS}

Partial funding by EPSRC Project Nos. EP/M029573/1 and EP/N024915/1 is gratefully acknowledged. C.P.N.C. acknowledges the Visiting Researcher fellowship provided by CAPES/INMETRO, Brazil facilitating her one year visit in UCL Mechanical Engineering, U.K.

\section{REFERENCES}

${ }^{1}$ N.-T. Nguyen and Z. Wu, "Micromixers-A review," J. Micromech. Microeng. 15, R1 (2005).

${ }^{2}$ A. Renfer, M. K. Tiwari, F. Meyer, T. Brunschwiler, B. Michel, and D. Poulikakos, "Vortex shedding from confined micropin arrays," Microfluid. Nanofluid. 15, 231 (2013).

${ }^{3}$ N. Cagney and S. Balabani, "Lagrangian structures and mixing in the wake of a streamwise oscillating cylinder," Phys. Fluids 28, 045107 (2016).

${ }^{4}$ A. Renfer, M. K. Tiwari, R. Tiwari, F. Alfieri, T. Brunschwiler, B. Michel, and D. Poulikakos, "Microvortex-enhanced heat transfer in 3D-integrated liquid cooling of electronic chip stacks," Int. J. Heat Mass Transfer 65, 33 (2013).

${ }^{5}$ B. Cantwell and D. Coles, "An experimental study of entrainment and transport in the turbulent near wake of a circular cylinder," J. Fluid Mech. 136, 321 (1983).

${ }^{6} \mathrm{~J}$. H. Gerrard, "The mechanics of the formation region of vortices behind bluff bodies," J. Fluid Mech. 25, 401 (1966).

${ }^{7}$ C. H. K. Williamson, "Vortex dynamics in the cylinder wake," Annu. Rev. Fluid Mech. 28, 477 (1996).

${ }^{8}$ V. M. Ribeiro, P. M. Coelho, F. T. Pinho, and M. A. Alves, "Three-dimensional effects in laminar flow past a confined cylinder," Chem. Eng. Sci. 84, 155 (2012).

${ }^{9}$ M. D. Griffith, J. Leontini, M. C. Thompson, and K. Hourigan, "Vortex shedding and three-dimensional behaviour of flow past a cylinder confined in a channel," J. Fluid Struct. 27, 855 (2011).

${ }^{10} \mathrm{M}$. Sahin and R. G. Owens, "A numerical investigation of wall effects up to high blockage ratios on two-dimensional flow past a confined circular cylinder," Phys. Fluids 16, 1305 (2004).

${ }^{11}$ S. Singha and K. P. Sinhamahapatra, "Flow past a circular cylinder between parallel walls at low Reynolds numbers," Ocean Eng. 37, 757 (2010).

${ }^{12}$ S. Turki, H. Abbassi, and S. B. Nasrallah, "Effect of the blockage ratio on the flow in a channel with a built-in square cylinder," Comput. Mech. 33, 22 (2003).

${ }^{13}$ R. W. Davis, E. F. Moore, and L. P. Purtell, "A numerical-experimental study of confined flow around rectangular cylinders," Phys. Fluids 27, 46 (1984).

${ }^{14} \mathrm{O}$. Inoue and A. Sakuragi, "Vortex shedding from a circular cylinder of finite length at low Reynolds numbers," Phys. Fluids 20, 033601 (2008).

${ }^{15}$ T. Lee and R. Budwig, "A study of the effect of aspect ratio on vortex shedding behind circular cylinders," Phys. Fluids 3, 309 (1991).

${ }^{16}$ A. Sohankar, C. Norberg, and L. Davidson, "Simulation of three-dimensional flow around a square cylinder at moderate Reynolds numbers," Phys. Fluids 11, 288 (1999).

${ }^{17}$ F. Rehimi, F. Aloui, S. B. Nasrallah, L. Doubliez, and J. Legrand, "Experimental investigation of a confined flow downstream of a circular cylinder centred between two parallel walls," J. Fluid Struct. 24, 855 (2008).

${ }^{18}$ J. Jung, C.-J. Kuo, Y. Peles, and M. Amitay, "The flow field around a micropillar confined in a microchannel," Int. J. Heat Fluid Flow 36, 118 (2012).

${ }^{19}$ M. Reyes, A. Velazquez, E. Martin, and J. R. Arias, "Experimental study on the confined 3D laminar flow past a square prism with a high blockage ratio," Int. J. Heat Fluid Flow 44, 444 (2013).

${ }^{20}$ A. Renfer, M. K. Tiwari, T. Brunschwiler, B. Michel, and D. Poulikakos, "Experimental investigation into vortex structure and pressure drop across microcavities in 3D integrated electronics," Exp. Fluids 51, 731 (2011). 
${ }^{21}$ S. M. Hagsäter, C. H. Westergaard, H. Bruus, and J. P. Kutter, "Investigations on LED illumination for micro-PIV including a novel front-lit configuration," Exp. Fluids 44, 211 (2008).

${ }^{22}$ J. M. Sherwood, D. Holmes, E. Kaliviotis, and S. Balabani, "Spatial distributions of red blood cells significantly alter local haemodynamics," PLoS One $\mathbf{9}$, e100473 (2014).

${ }^{23}$ B. J. Gemmell, H. Jiang, and E. J. Buskey, "A new approach to micro-scale particle image velocimetry $(\mu \mathrm{PIV})$ for quantifying flows around free-swimming zooplankton," J. Plankton Res. 36, 1396 (2014).

${ }^{24}$ M. Riccomi, F. Alberini, E. Brunazzi, and D. Vigolo, "Ghost particle velocimetry as an alternative to $\mu$ PIV for micro/milli-fluidic devices," Chem. Eng. Res. Des. 133, 183 (2018).

${ }^{25}$ A. Bamshad, A. Nikfarjam, and H. Khaleghi, "A new simple and fast thermallysolvent assisted method to bond PMMA-PMMA in micro-fluidics devices," J. Micromech. Microeng. 26, 065017 (2016).

${ }^{26}$ S. Pothos, A. Boomsma, D. Troolin, S. Bhattacharya, and P. Vlachos, Fora: Advances in Fluids Engineering Education, Cavitation and Multiphase Flow, Fluid Measurements and Instrumentation Vol. 2 (ASME, Washington, DC, USA, 2016), p. V002T10A005.

${ }^{27}$ M. G. Olsen and R. J. Adrian, "Out-of-focus effects on particle image visibility and correlation in microscopic particle image velocimetry," Exp. Fluids 29, S166 (2000).

${ }^{28}$ M. R. Rastan, A. Sohankar, and Md. M. Alam, "Low-Reynolds-number flow around a wall-mounted square cylinder: Flow structures and onset of vortex shedding," Phys. Fluids 29, 103601 (2017).

${ }^{29} \mathrm{H}$. Bruus, Theoretical Microfluidics (Oxford University Press, Oxford, New York, 2008).

${ }^{30}$ P. J. Strykowski and K. R. Sreenivasan, "On the formation and suppression of vortex 'shedding' at low Reynolds numbers,” J. Fluid Mech. 218, 71 (1990).
${ }^{31}$ F. H. Shair, A. S. Grove, E. E. Petersen, and A. Acrivos, "The effect of confining walls on the stability of the steady wake behind a circular cylinder," J. Fluid Mech. 17, 546 (1963).

${ }^{32}$ S. Balachandar, R. Mittal, and F. M. Najjar, "Properties of the mean recirculation region in the wakes of two-dimensional bluff bodies," J. Fluid Mech. 351, 167 (1997).

${ }^{33}$ U. Fey, M. König, and H. Eckelmann, "A new Strouhal-Reynolds-number relationship for the circular cylinder in the range $47<\operatorname{Re}<2 \times 10^{5}$," Phys. Fluids 10, 1547 (1998).

${ }^{34} \mathrm{C}$. Liang and G. Papadakis, "Large eddy simulation of cross-flow through a staggered tube bundle at subcritical Reynolds number," J. Fluid Struct. 23, 1215 (2007).

${ }^{35}$ S. Balabani and M. Yianneskis, "An experimental study of the mean flow and turbulence structure of cross-flow over tube bundles," Proc. Inst. Mech. Eng., Part C: J. Mech. Eng. Sci. 210, 317 (1996).

${ }^{36}$ F. Alfieri, M. K. Tiwari, A. Renfer, T. Brunschwiler, B. Michel, and D. Poulikakos, "Computational modeling of vortex shedding in water cooling of 3D integrated electronics," Int. J. Heat Fluid Flow 44, 745 (2013).

${ }^{37}$ S. C. Shadden, F. Lekien, and J. E. Marsden, "Definition and properties of Lagrangian coherent structures from finite-time Lyapunov exponents in two-dimensional aperiodic flows," Physica D 212, 271 (2005).

${ }^{38}$ D. Kadylak, P. Cave, and W. Mérida, "Effectiveness correlations for heat and mass transfer in membrane humidifiers," Int. J. Heat Mass Transfer 52, 1504 (2009).

${ }^{39}$ S. R. Gundlapally and V. Balakotaiah, "Heat and mass transfer correlations and bifurcation analysis of catalytic monoliths with developing flows," Chem. Eng. Sci. 66, $1879(2011)$ 